\title{
Sharply transitive sets in quasigroup actions
}

\author{
Bokhee Im • Ji-Young Ryu • Jonathan D.H. Smith
}

Received: 22 October 2009 / Accepted: 26 April 2010 / Published online: 19 May 2010

(C) Springer Science+Business Media, LLC 2010

\begin{abstract}
This paper forms part of the general development of the theory of quasigroup permutation representations. Here, the concept of sharp transitivity is extended from group actions to quasigroup actions. Examples of nontrivial sharply transitive sets of quasigroup actions are constructed. A general theorem shows that uniformity of the action is necessary for the existence of a sharply transitive set. The concept of sharp transitivity is related to two pairwise compatibility relations and to maximal cliques within the corresponding compatibility graphs.
\end{abstract}

Keywords Sharply transitive $\cdot$ Sharp transitivity $\cdot$ Simply transitive $\cdot$ Uniformly transitive · Permutation group · Permutation action · Group action · Quasigroup action $\cdot$ Lagrange Theorem $\cdot$ Permutation graph $\cdot$ Maximal clique

\section{Introduction}

A quasigroup is defined as a set $Q$ equipped with a multiplication, not necessarily associative, such that in the equation

$$
x \cdot y=z
$$

The first author was financially supported by Chonnam National University, 2009.

B. Im $(\bowtie) \cdot$ J.-Y. Ryu

Department of Mathematics, Chonnam National University, Gwangju 500-757, Republic of Korea e-mail: bim@chonnam.ac.kr

J.-Y. Ryu

e-mail: rjiyoung1004@hanmail.net

J.D.H. Smith

Department of Mathematics, Iowa State University, Ames, IA 50011, USA

e-mail: jdhsmith@math.iastate.edu 
knowledge of any two of the elements $x, y, z$ of $Q$ specifies the third uniquely. In particular, the solution for $x$ in terms of $y$ and $z$ is written as $z / y$. The body of the multiplication table of a finite quasigroup is a Latin square, while each Latin square may be bordered to give the multiplication table of a quasigroup structure on its set of entries. Nonempty associative quasigroups are groups. A large part of the study of quasigroups aims to extend various aspects of group theory to the more general context of quasigroups.

Groups arise from symmetry, understood through transitive group actions. Such actions may be defined equally well for quasigroups (where they lead to concepts of approximate symmetry). Let $P$ be a subquasigroup of a finite, nonempty quasigroup $Q$. The relative left multiplication group $\mathrm{LMlt}_{Q} P$ of $P$ in $Q$ is the subgroup of the symmetric group $Q$ ! on the set $Q$ which is generated by all the left multiplications

$$
L(p): Q \rightarrow Q ; \quad x \mapsto p x
$$

for elements $p$ of $P$. The homogeneous space $P \backslash Q$ is defined as the set of orbits of LMlt ${ }_{Q} P$ on $Q$. The cardinality $|P \backslash Q|$ is called the degree $d$ of the homogeneous space $P \backslash Q$. For each element $q$ of $Q$, the right multiplication by $q$ is the permutation

$$
R(q): Q \rightarrow Q ; \quad x \mapsto x q
$$

of $Q$. The action matrix $R_{P \backslash Q}(q)$ of $q$ on $P \backslash Q$ is the $d \times d$ row-stochastic matrix with entry

$$
\left[R_{P \backslash Q}(q)\right]_{X Y}=\frac{|X R(q) \cap Y|}{|X|}
$$

in the row labeled by the $\mathrm{LMlt}_{Q} P$-orbit $X$ and column labeled by the $\operatorname{LMlt}_{Q} P$ orbit $Y$. If $P$ is a subgroup of a finite group $Q$, then the homogeneous space $P \backslash Q$ is the set of right cosets of $P$, and the action matrices become permutation matrices. For further details, see [13].

The aim of the current paper is to extend the group-theoretic concept of sharp transitivity to the more general quasigroup case. The basic definition is presented in Sect. 2, and some direct consequences of the definition are recorded. Theorem 2.6 shows that uniformity of the homogeneous space is a necessary condition for the existence of a sharply transitive set.

Section 3 is devoted to the construction of two nontrivial sharply transitive sets of quasigroup actions and to an examination of their behavior from the standpoint of character theory. The constructions are conducted within the context of certain double-cover quasigroups for the group of symmetries of the equilateral triangle. These quasigroups are specified by directed graphs using the group as vertex set.

In the group case, sharp transitivity is known to correlate directly with the existence of large cliques in a compatibility graph $[13, \S 8.1] .{ }^{1}$ Section 4 introduces two distinct compatibility relations to study sharp transitivity in the quasigroup case: one

\footnotetext{
${ }^{1}$ For the natural actions of symmetric groups, Cameron-Ku [3] and Godsil-Meagher [4] have studied the maximal cocliques.
} 
weak, and one strong. The stronger condition implies the weaker. The two conditions coincide with the usual concept in the group case. In the strong compatibility graph, the existence of suitably large cliques is a sufficient condition for sharp transitivity (Theorem 4.7). On the other hand, Theorem 4.8 shows that the existence of large cliques in the weak compatibility graph is necessary for sharp transitivity. The example of Sect. 3 demonstrates that the weak and strong compatibility conditions really are distinct in the general quasigroup case.

\section{Sharp transitivity}

Definition 2.1 (Sharply transitive sets) Let $P$ be a subquasigroup of a finite, nonempty quasigroup $Q$. Then a subset $S$ of the set $\left\{R_{P \backslash Q}(q) \mid q \in Q\right\}$ of action matrices on the homogeneous space $P \backslash Q$ of degree $d$ is said to be sharply transitive if

$$
\sum_{R_{P \backslash Q}(q) \in S} R_{P \backslash Q}(q)=J_{d},
$$

with $J_{d}$ as the $d \times d$ all-ones matrix.

Remark 2.2 The quasigroup-theoretic concept of sharp transitivity introduced in Definition 2.1 specializes to the usual group-theoretic concept, as characterized by O'Nan [12]. Compare [13, Chap. 9, Exercise 17]. In Baer's original paper on the subject, sharp transitivity was called "simple transitivity" [1]. While placing sharp transitivity into the context of permutation geometries and matroids, Cameron used the term "uniform transitivity" [2].

Example 2.3 For a finite, nonempty quasigroup $Q$, the full set of action matrices of the regular homogeneous space $\varnothing \backslash Q$ forms a sharply transitive set [13, §4.4].

Proposition 2.4 Let $P$ be a nonempty subquasigroup of a finite quasigroup $Q$. Let $q$ be an element of $Q$. Then:

(a) The set $P$ forms a point of the homogeneous space $P \backslash Q$.

(b) For each point $X$ of the homogeneous space $P \backslash Q$, the action matrix entry $\left[R_{P \backslash Q}(q)\right]_{P X}$ is either 0 or 1 .

(c) There is a unique point $X$ such that $\left[R_{P \backslash Q}(q)\right]_{P X}=1$.

Proof (a) This follows since $P$ is a nonempty quasigroup. Recall that $p_{1} L\left(p_{2} / p_{1}\right)=$ $p_{2}$ for elements $p_{1}$ and $p_{2}$ of $P$ with $p_{2} / p_{1}$ in $P$.

(b) Suppose that the action matrix entry $\left[R_{P \backslash Q}(q)\right]_{P X}$ is nonzero, so there are elements $p_{0}$ of $P$ and $x$ of $X$ such that $p_{0} q=x$. In particular,

$$
q=x L\left(p_{0}\right)^{-1} \text {. }
$$

Then for each element $p$ of $P$, we have

$$
p q=x L\left(p_{0}\right)^{-1} L(p) \in x \operatorname{LMlt}_{Q}(P)=X,
$$


so $\left[R_{P \backslash Q}(q)\right]_{P X}=1$.

(c) This follows since the action matrices are row-stochastic.

Proposition 2.5 Let $P$ be a nonempty subquasigroup of a finite quasigroup $Q$. Let $S$ be a sharply transitive set of action matrices on the homogeneous space $P \backslash Q$ of degree d. Then:

(a) For each point $X$ of $P \backslash Q$, the sharply transitive set $S$ comprises exactly one action matrix $R_{P \backslash Q}(q)$ with $\left[R_{P \backslash Q}(q)\right]_{P X}=1$ (and $\left[R_{P \backslash Q}(q)\right]_{P Y}=0$ for points $Y$ distinct from $X)$.

(b) The cardinality of $S$ is the degree $d$ of the homogeneous space.

Proof

(a) Apply Proposition 2.4.

(b) This follows from (a).

Recall that a subquasigroup $P$ of a finite, nonempty quasigroup $Q$ is said to be (right) Lagrangean if $\mathrm{LMlt}_{Q} P$ acts semitransitively, so that its orbits all have the same cardinality [13, §4.5]. In this context, the homogeneous space $P \backslash Q$ is said to be uniform.

Theorem 2.6 Let $P$ be a subquasigroup of a finite, nonempty quasigroup $Q$. If there is a sharply transitive set of action matrices on the homogeneous space $P \backslash Q$, then $P$ is Lagrangean.

Proof Since LMlt $Q \varnothing$ acts semitransitively, $\varnothing$ is Lagrangean. Indeed, Example 2.3 notes that the regular homogeneous space $\varnothing \backslash Q$ admits a sharply transitive set. Now suppose that $P$ is nonempty and non-Lagrangean. In view of Proposition 2.4(a), the set $P$ forms a point of $P \backslash Q$. Suppose that there is a sharply transitive set $S$ of action matrices. By Proposition 2.5(b), the cardinality of $S$ is the degree $d$ of $P \backslash Q$. Then since

$$
\sum_{X \in P \backslash Q}\left[J_{d}\right]_{X P}=d,
$$

the equation

$$
\sum_{X \in P \backslash Q} \sum_{R_{P \backslash Q}(q) \in S}\left[R_{P \backslash Q}(q)\right]_{X P}=d
$$

follows by the sharp transitivity (2.1).

For each element $x$ of $Q$, the orbit $x \operatorname{LMlt}_{Q} P$ includes the set

$$
\{x L(p) \mid p \in P\}
$$

of $|P|$ distinct elements. Thus for each point $X$ of $P \backslash Q$, the inequality

$$
|P| \leq|X|
$$


holds. Furthermore, since $P$ is not Lagrangean, there is at least one point $X$ for which the inequality is strict.

Now consider the action matrix $R_{P \backslash Q}(q)$ of an element $q$ in $Q$. For each point $X$ of $P \backslash Q$, we have

$$
\left[R_{P \backslash Q}(q)\right]_{X P}=\frac{|X R(q) \cap P|}{|X|} \leq \frac{|X R(q) \cap P|}{|P|} .
$$

Moreover, when $|P|<|X|$ and $|X R(q) \cap P| \neq 0$, we have

$$
\left[R_{P \backslash Q}(q)\right]_{X P}<\frac{|X R(q) \cap P|}{|P|} .
$$

Thus,

$$
\begin{aligned}
\sum_{X \in P \backslash Q}\left[R_{P \backslash Q}(q)\right]_{X P} & \leq \sum_{X \in P \backslash Q} \frac{|X R(q) \cap P|}{|P|} \\
& =\frac{1}{|P|} \sum_{X \in P \backslash Q}|X R(q) \cap P|=\frac{|P|}{|P|}=1,
\end{aligned}
$$

the penultimate equality holding since the subsets $X R(q) \cap P$ of $P$ form a partition of $P$ as $X$ ranges over $P \backslash Q$. Moreover,

$$
\sum_{X \in P \backslash Q}\left[R_{P \backslash Q}(q)\right]_{X P}<1
$$

if $|X R(q) \cap P| \neq 0$ for an orbit $X$ with $|P|<|X|$.

By (2.1), the sharply transitive set $S$ includes the action matrix $R_{P \backslash Q}(q)$ of an element $q$ of $Q$ with $|X R(q) \cap P| \neq 0$ for an orbit $X$ with $|P|<|X|$. Indeed, the matrix on the left-hand side of (2.1) must have a nonzero entry in the row labeled $X$ and column labeled $P$. By the considerations of the preceding paragraph, it follows that

$$
\sum_{R \backslash Q} \sum_{X) \in S}\left[R_{P \backslash Q}(q)\right]_{X P}<d .
$$

This inequality contradicts (2.2).

Corollary 2.7 Suppose that $P$ is a 2-element subquasigroup of a finite quasigroup $Q$. If

$$
\left\{R_{P \backslash Q}(q) \mid q \in S\right\}
$$

is a sharply transitive set of action matrices on $P \backslash Q$, then the complementary set

$$
\left\{R_{P \backslash Q}(q) \mid q \in Q \backslash S\right\}
$$

is also sharply transitive. 
Proof Let $|Q|=n$. By Theorem 2.6, $P$ is Lagrangean in $Q$. Then

$$
\sum_{q \in Q} R_{P \backslash Q}(q)=2 J_{n / 2}
$$

by [13, Corollary $4.2(a)]$. Since (2.3) is sharply transitive, one has

$$
\sum_{q \in S} R_{P \backslash Q}(q)=J_{n / 2} .
$$

From (2.5) one then obtains the equation

$$
\sum_{q \in Q \backslash S} R_{P \backslash Q}(q)=J_{n / 2},
$$

showing that (2.4) is sharply transitive.

\section{Double covers of the symmetric group}

In order to exhibit nontrivial sharply transitive sets of quasigroup action matrices, we will consider quasigroups obtained by permuting entries in the multiplication table of the direct product $S_{3} \times \mathbb{Z} / 2$ of the symmetric group $\{0,1,2\}$ ! with the two-element additive group $\{0,1\}$ of integers modulo 2 . To establish a convenient notation, define the rotations (numbered by the final element in the cycle starting at 0 )

$$
\rho_{0}=(0), \quad \rho_{1}=\left(\begin{array}{lll}
0 & 2 & 1
\end{array}\right), \quad \rho_{2}=\left(\begin{array}{lll}
0 & 1 & 2
\end{array}\right)
$$

and reflexions ("Spiegelungen," numbered by the fixed point)

$$
\sigma_{0}=\left(\begin{array}{ll}
1 & 2
\end{array}\right), \quad \sigma_{1}=\left(\begin{array}{ll}
0 & 2
\end{array}\right), \quad \sigma_{2}=\left(\begin{array}{ll}
0 & 1
\end{array}\right) .
$$

For an element $\pi$ of $S_{3}$, write $\pi^{0}$ for the pair $(\pi, 0)$ in the product and $\pi^{1}$ for the pair $(\pi, 1)$. Consider a relation $\lambda \cdot \mu=v$ in $S_{3}$. The corresponding fragment

$$
\left[\begin{array}{ll}
v^{0} & v^{1} \\
v^{1} & v^{0}
\end{array}\right]
$$

of the body of the multiplication table of $S_{3} \times \mathbb{Z} / 2$, indexed by the respective rows labeled $\lambda^{0}, \lambda^{1}$ and columns labeled $\mu^{0}, \mu^{1}$, is known as the intercalate ${ }^{2}$ corresponding to the source $\lambda$ and sink $\mu$.

The body of the multiplication table of the group $S_{3} \times \mathbb{Z} / 2$ is a Latin square. It remains a Latin square if the intercalate (3.1) is changed to

$$
\left[\begin{array}{ll}
v^{1} & v^{0} \\
v^{0} & v^{1}
\end{array}\right]
$$

\footnotetext{
${ }^{2}$ Compare [5, 11]; the term was introduced in [10]. Note that $S_{3} \times \mathbb{Z} / 2$ also includes other intercalates that are internal to the copies of $S_{3}$. 
Fig. 1 The directed graph $\Gamma$ on $S_{3}$

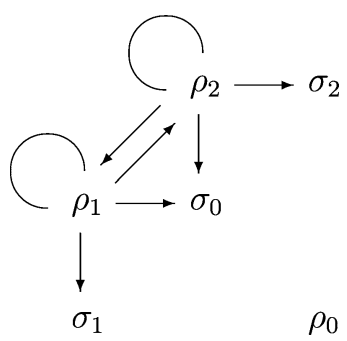

Altogether, $2^{36}$ quasigroup structures on the set $S_{3} \times \mathbb{Z} / 2$ are obtained by making such intercalate changes in the body of the multiplication table of the group $S_{3} \times \mathbb{Z} / 2$. Each such quasigroup is specified uniquely by a directed graph $\Gamma$ (in which loops are allowed) on the vertex set $S_{3}$ : The intercalate corresponding to the source $\lambda$ and sink $\mu$ is changed precisely when there is a directed edge in $\Gamma$ from the source $\lambda$ to the sink $\mu$. Write $Q(\Gamma)$ for the quasigroup specified in this way by a directed graph $\Gamma$. Let $\left[\gamma_{\lambda \mu}\right]$ be the adjacency matrix of $\Gamma$, interpreted as a matrix over the ring $\mathbb{Z} / 2$. Then for $\lambda, \mu$ in $S_{3}$ and $l, m$ in $\mathbb{Z} / 2$, the equation

$$
\lambda^{l} \cdot \mu^{m}=v^{l+m+\gamma \lambda \mu}
$$

specifies the product of $\lambda^{l}$ and $\mu^{m}$ in $Q(\Gamma)$, given the product relation $\lambda \cdot \mu=v$ in $S_{3}$. Note that the projection

$$
\theta: Q(\Gamma) \rightarrow S_{3} ; \quad \lambda^{l} \mapsto \lambda
$$

is a quasigroup homomorphism.

For nontrivial sharply transitive sets, consider the directed graph $\Gamma$ on the vertex set $S_{3}$ given by Fig. 1 . The corresponding quasigroup $Q=Q(\Gamma)$ has a subquasigroup $P=\left\{\rho_{0}^{0}, \sigma_{0}^{0}\right\}$, since $\rho_{0}$ and $\sigma_{0}$ are not adjacent in $\Gamma$. The homogeneous space $P \backslash Q$ is

$$
\left\{\left\{\rho_{0}^{0}, \sigma_{0}^{0}\right\},\left\{\rho_{0}^{1}, \sigma_{0}^{1}\right\},\left\{\rho_{1}^{0}, \sigma_{1}^{0}\right\},\left\{\rho_{1}^{1}, \sigma_{1}^{1}\right\},\left\{\rho_{2}^{0}, \sigma_{2}^{0}\right\},\left\{\rho_{2}^{1}, \sigma_{2}^{1}\right\}\right\}
$$

Note that in the general context of this section, the space $P \backslash Q$ is determined by the neighborhood of $P$, the set of edges in $\Gamma$ whose source lies in the image $P^{\theta}$ of $P$ under the projection (3.3). In Fig. 1, this neighborhood is empty.

Define $2 \times 2$ matrices

$$
O=\left[\begin{array}{ll}
0 & 0 \\
0 & 0
\end{array}\right], \quad I=\left[\begin{array}{ll}
1 & 0 \\
0 & 1
\end{array}\right], \quad T=\left[\begin{array}{ll}
0 & 1 \\
1 & 0
\end{array}\right], \quad U=\left[\begin{array}{ll}
1 / 2 & 1 / 2 \\
1 / 2 & 1 / 2
\end{array}\right],
$$

the respective zero, identity, transposition, and uniform matrices. With the six points of $P \backslash Q$ ordered as in (3.4), the action matrices of the quasigroup elements $\rho_{i}^{l}$, for 
Fig. 2 Character table of $Q(\Gamma)$

SHARPLY TRANSITIVE QUASIGROUP ACTIONS

\begin{tabular}{c|cccc} 
& 1 & 1 & 4 & 6 \\
\hline$Q(\Gamma)$ & $\rho_{0}^{0}$ & $\rho_{0}^{1}$ & $\rho_{+}^{*}$ & $\sigma_{*}^{*}$ \\
\hline$\psi_{1}$ & 1 & 1 & 1 & 1 \\
$\psi_{-1}$ & 1 & 1 & 1 & -1 \\
$\psi_{2}$ & 2 & 2 & -1 & 0 \\
$\psi_{3}$ & $\sqrt{6}$ & $-\sqrt{6}$ & 0 & 0 \\
\hline$\psi_{1}+\psi_{2}+\psi_{3} \cdot \sqrt{3 / 2}=\pi$ & 6 & 0 & 0 & 1
\end{tabular}

$0 \leq i \leq 2$ and $0 \leq l \leq 1$, form the sharply transitive set

$$
\left\{\begin{array}{lll}
{\left[\begin{array}{lll}
I & O & O \\
O & I & O \\
O & O & I
\end{array}\right],} & {\left[\begin{array}{lll}
T & O & O \\
O & T & O \\
O & O & T
\end{array}\right],} & {\left[\begin{array}{lll}
O & I & O \\
O & O & U \\
U & O & O
\end{array}\right],} \\
{\left[\begin{array}{lll}
O & T & O \\
O & O & U \\
U & O & O
\end{array}\right],} & {\left[\begin{array}{lll}
O & O & I \\
U & O & O \\
O & U & O
\end{array}\right],} & {\left[\begin{array}{lll}
O & O & T \\
U & O & O \\
O & U & O
\end{array}\right]}
\end{array}\right.
$$

of block matrices. The action matrices of the quasigroup elements $\sigma_{i}^{l}$, for $0 \leq i \leq 2$ and $0 \leq l \leq 1$, then form the complementary set

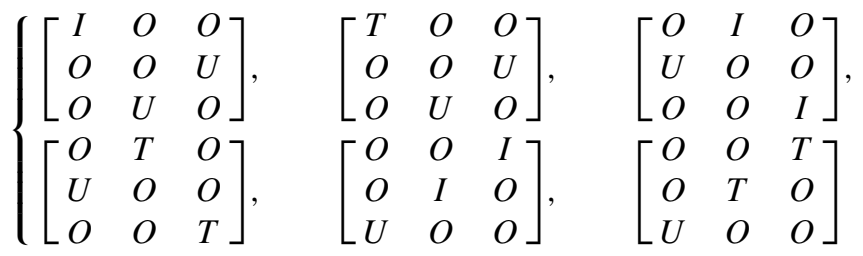

of block matrices, sharply transitive in accordance with Corollary 2.7.

It is instructive to consider these examples from the standpoint of character theory. The character table of $Q(\Gamma)$ is presented in Fig. 2. In order to stay as close as possible to the more familiar group case, we consider $Q(\Gamma)$ as a pique with pointed idempotent $\rho_{0}^{0}$ (compare [13, pp. 9, 141-142]).

Note that the stabilizer of the pointed idempotent $\rho_{0}^{0}$ within the multiplication group of $Q(\Gamma)$ has orbits (pique conjugacy classes)

$$
\left\{\rho_{0}^{0}\right\}, \quad\left\{\rho_{0}^{1}\right\}, \quad\left\{\rho_{1}^{0}, \rho_{1}^{1}, \rho_{2}^{0}, \rho_{2}^{1}\right\}, \quad\left\{\sigma_{0}^{0}, \sigma_{1}^{0}, \sigma_{2}^{0}, \sigma_{0}^{1}, \sigma_{1}^{1}, \sigma_{2}^{1}\right\} .
$$

These four conjugacy classes are denoted respectively as $\rho_{0}^{0}, \rho_{0}^{1}, \rho_{+}^{*}, \sigma_{*}^{*}$ in the table. The first three basic characters $\psi_{1}, \psi_{-1}, \psi_{2}$ are the lifts to $Q(\Gamma)$ of the irreducible characters of $S_{3}$, obtained by application of the Quotient Theorem to the homomorphism $\theta$ of (3.3) [8], [13, Theorem 7.2]. The fourth basic character $\psi_{3}$ of $Q(\Gamma)$ is then given by the orthogonality relations [6], [13, Theorem 6.6]. For convenience, the valencies $n_{i}$-compare [6], [13, (6.23)]—are listed in the top row of Fig. 2. 
The bottom row of Fig. 2 gives the permutation character $\pi$ of the homogeneous space $P \backslash Q$, computed as in [9], [13, (9.23)]. Note that in this case, $\pi$ does coincide with the identity character of $P$ induced up to $Q$. (For induction, compare [7], $[13, \S 7.4]$. For an example of a permutation character that is not obtained by inducing up from the "stabilizer," compare [13, Remark 9.2].)

The nonprincipal basic summands of $\pi$ are $\psi_{2}$ and $\psi_{3}$, the latter with multiplicity $\sqrt{3 / 2}$. For each of these nonprincipal basic summands $\psi$, O'Nan's group-theoretical condition

$$
\sum_{s \in S} \psi(s)=0
$$

[12], [13, Example 9.17(b)] holds for each of the sets

$$
S=\left\{\rho_{i}^{j} \mid 0 \leq i \leq 2,0 \leq j \leq 1\right\}
$$

and

$$
S=\left\{\sigma_{i}^{j} \mid 0 \leq i \leq 2,0 \leq j \leq 1\right\}
$$

corresponding to the complementary sharply transitive sets of (3.5) and (3.6).

As a final observation, note that the traces of the action matrices in (3.6) take the distinct values 2 and 0 , even though the matrices all represent actions from the single conjugacy class $\sigma_{*}^{*}$. (Recall that in the group case, conjugate elements have similar permutation matrices.) The common permutation character value $\pi\left(\sigma_{i}^{j}\right)=1$, for $0 \leq i \leq 2$ and $j=0,1$, is the arithmetic mean of the traces, taken over all six members of the conjugacy class $\sigma_{*}^{*}$. The possible variation of the traces of action matrices within a given conjugacy class is one of the obstacles impeding attempts to extend O'Nan's criterion (3.7) beyond the group case.

\section{Compatibility}

Definition 4.1 (Strong and weak compatibility) Suppose that $P$ is a subquasigroup of a finite, nonempty quasigroup $Q$.

(a) Two distinct elements $q_{1}$ and $q_{2}$ of $Q$ are said to be strongly compatible (in the action on $P \backslash Q$ ) if

$$
R\left(q_{1}\right)^{-1}(Y) \cap R\left(q_{2}\right)^{-1}(Y)=\varnothing
$$

for all points $Y$ of $P \backslash Q$.

(b) Two distinct elements $q_{1}$ and $q_{2}$ of $Q$ are said to be weakly compatible (in the action on $P \backslash Q)$ if

$$
\left[R_{P \backslash Q}\left(q_{1}\right)\right]_{X Y}+\left[R_{P \backslash Q}\left(q_{2}\right)\right]_{X Y} \leq 1
$$

for all points $X$ and $Y$ of $P \backslash Q$.

(c) The strong compatibility graph of the space $P \backslash Q$ is the undirected graph (without loops), on the vertex set $Q$, in which two vertices are joined by an edge if and only if they are strongly compatible in the action of $Q$ on $P \backslash Q$. 
Fig. 3 The complement of the weak compatibility graph for the homogeneous space $\left\{\rho_{0}^{0}, \sigma_{0}^{0}\right\} \backslash Q(\Gamma)$

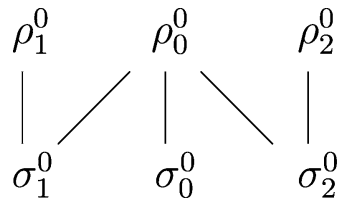

(d) The weak compatibility graph of the space $P \backslash Q$ is the undirected graph (without loops), on the vertex set $Q$, in which two vertices are joined by an edge if and only if they are weakly compatible in the action of $Q$ on $P \backslash Q$.

Remark 4.2 The strong and weak compatibilities of Definition 4.1 both coincide with the usual concept in the group case $[13, \S 8.1]$.

Proposition 4.3 Let $P$ be a subquasigroup of a finite, nonempty quasigroup $Q$. In the action on $P \backslash Q$, the strong compatibility of two elements $q_{1}, q_{2}$ of $Q$ implies their weak compatibility.

Proof Consider points $X$ and $Y$ of $P \backslash Q$. By Definition 4.1(a), we have

$$
R\left(q_{1}\right)^{-1}(Y) \cap R\left(q_{2}\right)^{-1}(Y)=\varnothing,
$$

so

$$
\left(X \cap R\left(q_{1}\right)^{-1}(Y)\right) \cap\left(X \cap R\left(q_{2}\right)^{-1}(Y)\right)=\varnothing .
$$

Since $X \cap R\left(q_{1}\right)^{-1}(Y)$ and $X \cap R\left(q_{2}\right)^{-1}(Y)$ are disjoint subsets of $X$, it follows that

$$
\left|X \cap R\left(q_{1}\right)^{-1}(Y)\right|+\left|X \cap R\left(q_{2}\right)^{-1}(Y)\right| \leq|X| .
$$

Dividing by $|X|$ and rewriting the numerators of the resulting fractions yields

$$
\frac{\left|X R\left(q_{1}\right) \cap Y\right|}{|X|}+\frac{\left|X R\left(q_{2}\right) \cap Y\right|}{|X|} \leq 1,
$$

as required to satisfy the condition of Definition 4.1(b).

Corollary 4.4 The edge set of the strong compatibility graph of $P \backslash Q$ is a subset of the edge set of the weak compatibility graph of $P \backslash Q$. In particular, if a subset of $Q$ forms a clique in the strong compatibility graph, then it also forms a clique in the weak compatibility graph.

Figure 3 displays the bipartite complement of the weak compatibility graph for the action $P \backslash Q$ of Sect. 3. The top part corresponds to the sharply transitive set (3.5), while the bottom part corresponds to the sharply transitive set (3.6).

Proposition 4.5 Let $P$ be a subgroup of a finite group $Q$. In the action on $P \backslash Q$, the strong compatibility of two elements $q_{1}, q_{2}$ of $Q$ is equivalent to their weak compatibility. 
Proof By Proposition 4.3, it suffices to show that weak compatibility implies strong compatibility in this case. Let $q_{1}$ and $q_{2}$ be weakly, but not strongly compatible. Thus there is some point $Y$ of $P \backslash Q$ with

$$
R\left(q_{1}\right)^{-1}(Y) \cap R\left(q_{2}\right)^{-1}(Y) \neq \varnothing,
$$

say $x q_{1} \in Y$ and $x q_{2} \in Y$ for some element $x$ of $Q$. Then for $X=P x$, we have $X q_{1}=Y=X q_{2}$. This yields the contradiction

$$
\left[R_{P \backslash Q}\left(q_{1}\right)\right]_{X Y}+\left[R_{P \backslash Q}\left(q_{2}\right)\right]_{X Y}=2
$$

to the weak compatibility of $q_{1}$ and $q_{2}$.

Proposition 4.6 Let $P$ be a subquasigroup of a general finite nonempty quasigroup $Q$. In the action on $P \backslash Q$, the weak compatibility of two elements $q_{1}, q_{2}$ of $Q$ does not necessarily imply their strong compatibility.

Proof Consider the quasigroup $Q(\Gamma)$ specified by the graph of Fig. 1, with its subquasigroup $P=\left\{\rho_{0}^{0}, \sigma_{0}^{0}\right\}$. Since

$$
R_{P \backslash Q}\left(\rho_{2}^{0}\right)=\left[\begin{array}{ccc}
O & O & I \\
U & O & O \\
O & U & O
\end{array}\right] \text { and } R_{P \backslash Q}\left(\sigma_{1}^{0}\right)=\left[\begin{array}{ccc}
O & I & O \\
U & O & O \\
O & O & I
\end{array}\right],
$$

the elements $\rho_{2}^{0}$ and $\sigma_{1}^{0}$ are weakly compatible. On the other hand, (3.2) shows that

$$
\rho_{1}^{1} \rho_{2}^{0}=\rho_{0}^{0} \in P \quad \text { and } \quad \rho_{1}^{1} \sigma_{1}^{0}=\sigma_{0}^{0} \in P,
$$

so the elements $\rho_{2}^{0}$ and $\sigma_{1}^{0}$ are not strongly compatible.

Theorem 4.7 (Cliques within the strong compatibility graph) Let $P$ be a Lagrangean subquasigroup of a finite, nonempty quasigroup $Q$. Let the degree of $P \backslash Q$ be $d$. Suppose that a d-element subset

$$
K=\left\{q_{1}, \ldots, q_{d}\right\}
$$

of $Q$ forms a clique in the strong compatibility graph of $P \backslash Q$. Then:

(a) The clique $K$ is maximal in the strong compatibility graph.

(b) The set

$$
S=\left\{R_{P \backslash Q}\left(q_{1}\right), \ldots, R_{P \backslash Q}\left(q_{d}\right)\right\}
$$

of action matrices is sharply transitive.

Proof (a) Since $P$ is Lagrangean, each point $Y$ of $P \backslash Q$ has size $|Q| / d$. Now as $q_{i}$ ranges over the vertex set of the $d$-element clique $K$ in the strong compatibility graph, the $d$ mutually disjoint $(|Q| / d)$-element subsets $R\left(q_{i}\right)^{-1}(Y)$ of $Q$ cover $Q$, so that

$$
Q=\bigcup_{q_{i} \in K} R\left(q_{i}\right)^{-1}(Y)
$$


If an element $q$ of $Q$ lies in the complement of $K$, then $R(q)^{-1}(Y)$ intersects nontrivially with some $R\left(q_{i}\right)^{-1}(Y)$ for $q_{i}$ in $K$. Since $q$ and $q_{i}$ are not adjacent in the strong compatibility graph, the clique $K$ is maximal.

(b) Using (4.1), each point $X$ of $P \backslash Q$ is expressed as

$$
\begin{aligned}
X & =X \cap Q=X \cap \bigcup_{q_{i} \in K} R\left(q_{i}\right)^{-1}(Y) \\
& =\bigcup_{q_{i} \in K}\left(X \cap R\left(q_{i}\right)^{-1}(Y)\right),
\end{aligned}
$$

a disjoint union. Thus,

$$
\sum_{q_{i} \in K}\left[R_{P \backslash Q}\left(q_{i}\right)\right]_{X Y}=\sum_{q_{i} \in K} \frac{\left|X \cap R\left(q_{i}\right)^{-1} Y\right|}{|X|}=1,
$$

as required to show that $S$ is sharply transitive.

Theorem 4.8 (Cliques in the weak compatibility graph) Let $P$ be a subquasigroup of a finite, nonempty quasigroup $Q$. Then a subset

$$
K=\left\{q_{1}, \ldots, q_{k}\right\}
$$

of $Q$ forms a maximal clique in the weak compatibility graph of $P \backslash Q$ if the set

$$
S=\left\{R_{P \backslash Q}\left(q_{1}\right), \ldots, R_{P \backslash Q}\left(q_{k}\right)\right\}
$$

of action matrices is sharply transitive.

Proof Suppose that $S$ is sharply transitive. It will first be noted that $K$ forms a clique in the weak compatibility graph. Indeed, if two elements $q_{i}$ and $q_{j}$ of $K$ are not adjacent, then there are points $X$ and $Y$ of $P \backslash Q$ such that

$$
\left[R_{P \backslash Q}\left(q_{1}\right)\right]_{X Y}+\left[R_{P \backslash Q}\left(q_{2}\right)\right]_{X Y}>1 .
$$

This would imply

$$
\sum_{R_{P \backslash Q}(q) \in S}\left[R_{P \backslash Q}(q)\right]_{X Y}>\left[J_{d}\right]_{X Y},
$$

in violation of condition (2.1) for sharp transitivity.

If $P$ is empty, then $S$ is the full set of all action matrices, as observed in Example 2.3. In this case, the clique $Q$ is certainly maximal. For the remainder of the proof, we may assume that $P$ is nonempty and that $K$ is a proper subset of $Q$. Consider an element $q$ from the complement of $K$ in $Q$. By Proposition 2.4(c), there is a point $X$ of $P \backslash Q$ with $\left[R_{P \backslash Q}(q)\right]_{P X}=1$. But by Proposition 2.5(a), there is an element $q_{i}$ of $K$ with $\left[R_{P \backslash Q}\left(q_{i}\right)\right]_{P X}=1$. Since

$$
\left[R_{P \backslash Q}(q)\right]_{P X}+\left[R_{P \backslash Q}\left(q_{i}\right)\right]_{P X}=2,
$$


the vertices $q$ and $q_{i}$ are not weakly compatible. This means that the clique $K$ is maximal.

Acknowledgements We are grateful to the editor and referees for many helpful comments.

\section{References}

1. Baer, R.: Nets and groups I. Trans. Am. Math. Soc. 46, 110-141 (1939)

2. Cameron, P.J.: Metric and geometric properties of sets of permutations. In: Deza, M.-M., Frankl, P., Rosenberg, I.G. (eds.) Algebraic, Extremal and Metric Combinatorics 1986. pp. 39-53. Cambridge University Press, Cambridge (1988)

3. Cameron, P.J., Ku, C.Y.: Intersecting families of permutations. Eur. J. Comb. 24, 881-890 (2003)

4. Godsil, C., Meagher, K.: A new proof of the Erdős-Ko-Rado theorem for intersecting families of permutations. arXiv:0710.2109v1

5. Heinrich, K., Wallis, W.D.: The maximum number of intercalates in a Latin square. In: McAvaney, K.L. (ed.) Combinatorial Mathematics VIII, pp. 221-233. Springer, Berlin (1981)

6. Johnson, K.W., Smith, J.D.H.: Characters of finite quasigroups. Eur. J. Comb. 5, $43-50$ (1984)

7. Johnson, K.W., Smith, J.D.H.: Characters of finite quasigroups II: induced characters. Eur. J. Comb. 7, 131-137 (1986)

8. Johnson, K.W., Smith, J.D.H.: Characters of finite quasigroups III: quotients and fusion. Eur. J. Comb. 10, 47-56 (1989)

9. Johnson, K.W., Smith, J.D.H.: Characters of finite quasigroups VII: permutation characters. Comment. Math. Univ. Carol. 45, 265-273 (2004)

10. Norton, H.W.: The $7 \times 7$ squares. Ann. Eugen. 9, 269-307 (1939)

11. McKay, B.D., Wanless, I.M.: Most Latin squares have many subsquares. J. Comb. Theory, Ser. A 86, 322-347 (1999)

12. O'Nan, M.E.: Sharply 2-transitive sets of permutations. In: Aschbacher, M., et al. (eds.) Proceedings of the Rutgers Group Theory Year, 1983-1984, pp. 63-67. Cambridge University Press, Cambridge (1984)

13. Smith, J.D.H.: An Introduction to Quasigroups and Their Representations. Chapman and Hall/CRC, Boca Raton (2007) 